\title{
Agreement of Networks of Discrete-Time Agents with Mixed Dynamics and Time Delays
}

\author{
Xue Li, Xueer Chen, and Yingchun Xie \\ School of Aeronautics and Astronautics, University of Electronic Science and Technology of China, Chengdu 611731, China \\ Correspondence should be addressed to Xue Li; lixue.1981@uestc.edu.cn
}

Received 17 October 2015; Accepted 29 November 2015

Academic Editor: Peng Lin

Copyright (C) 2015 Xue Li et al. This is an open access article distributed under the Creative Commons Attribution License, which permits unrestricted use, distribution, and reproduction in any medium, provided the original work is properly cited.

\begin{abstract}
This paper considers agreement problems of networks of discrete-time agents with mixed dynamics and arbitrary bounded time delays, and networks consist of first-order agents and second-order agents. By using the properties of nonnegative matrices and model transformations, we derive sufficient conditions for stationary agreement of networks with bounded time delays. It is shown that stationary agreement can be achieved with arbitrary bounded time delays, if and only if fixed topology has a spanning tree and the union of the dynamically changing topologies has a spanning tree. Simulation results are also given to demonstrate the effectiveness of our theoretical results.
\end{abstract}

\section{Introduction}

Agreement problems for multiagent networks have attracted great attention in recent years. Agreement means that multiple agents can reach a common value with time going, which might be attitude in multiple spacecraft alignment, heading direction in flocking behaviour, or average in distributed computation. In several decades, numerous literatures have studied the agreement problems for multiagent networks [117]. In [1], by using Lyapunov function method and LaSalle's invariance principle, the agreement problems can be solved when the communication topologies were undirected connected graphs and leader-following network. And in [2], the authors studied the stability properties of linear time-varying networks in continuous time whose network matrix was Mezter with zero row sums and provided that the delay only affects the off-diagonal terms in the differential equation.

Because of the noise, packet loss, and limited communication bandwidth, time delays are inevitable when agents send and receive the information from their neighbors. Agreement problems for the continuous-time multiagent networks with time delays have attracted great attention in the past decades [2-8]. In [3], the authors addressed a coordination problem of a multiagent network with jointly connected interconnection topologies, a sufficient condition to make all the agents converge to a common value based on Lyapunov-based approach and related space decomposition technique. In [4], based on reduced-order system, the authors presented conditions under which all agents reach consensus with the desired $H_{\infty}$ performance. In addition, agreement problems for the discrete-time multiagent networks with time delays have also attracted great attention in the past decades [9-12]. In [9], the authors investigated the agreement problem of second-order discrete-time multiagent networks with nonuniform time delays and changing communication topologies, and by using the properties of nonnegative matrices, all agents reached agreement with arbitrary bounded nonuniform time delays. In $[10]$, the authors extended the results of $[9,11]$ by proposing a new method based on an undelayed equivalent network which has two parts: the linear main body and the error auxiliary, and it was shown that the network can reach agreement with arbitrary communication delays. In [18], the authors investigated a systematical framework of agreement problems with directed interconnection graphs or time delays by a Lyapunov-based approach. In this section, we want to pay more attention to mixed multiagent networks with time delays $[19,20]$. In [19], the author obtained two agreement conditions by using the properties of nonnegative matrix. 
In [20], by using frequency domain method and Gershgorin disk theorem, agreement of the protocol with time delay was only dependent on network coupling strength and each input time delay, but independent of communication delay.

The purpose of this work is to extend the method based on the nonnegative matrices $[9,11,19,21,22]$ to the discretetime agents with mixed dynamics and mixed orders. We first introduce two different linear agreement protocols of secondorder network and first-order network, respectively. Then, by model transformations, we turn the original network into an equivalent undelayed network whose network matrix is stochastic. Compared with the works $[9,19]$, we use much easier dynamics and two different protocols with varying control parameters. Under some restrictions on the sampling interval and the coupling weights, we obtain important results. Agreement of two different protocols with time delays is only dependent on the connectedness of the interconnection topology, but independent of communication time delays.

In this paper, the following notation will be used. $R^{m}$ denotes the set of all $m$-dimensional real column vectors; $Z_{+}$represents the set of nonnegative integers; $\mathbf{1}_{n}$ represents $[1,1, \ldots, 1]^{T}$ with $n$ dimension; $I_{n}$ denotes $n \times n$ identity matrix; 0 denotes a zero value or zero matrix with an appropriate dimension; $M_{n}(R)$ represents the set of all $n \times n$ real matrices.

\section{Preliminaries}

In this section, we give some preliminary knowledge about matrix theory and graph theory. Let $\varsigma=(V, E, A)$ be a weighted digraph, where $V=\left\{v_{1}, \ldots, v_{n}\right\}$ is the set of $n$ nodes and $E \subseteq V \times V$ is the set of edges. $I=\{1,2, \ldots, n\}$ is the set of node indexes. An edge of $E$ is denoted by $e_{i j}=\left(v_{i}, v_{j}\right)$, where the first element $v_{i}$ of $e_{i j}$ is said to be the tail of the edge and the other $v_{j}$ to be the head. $A=\left[a_{i j}\right]$ is a weighted adjacency matrix, where $a_{i j} \geq 0$ denotes the weight of $v_{i}$ to $v_{j}$. If $a_{i j}>0$, this means that the node can obtain information from the node; if $a_{i j}=0$, this means that the node cannot obtain information from the node. Define the set of neighbors of node $i$ as $N_{i}=\left\{j: a_{i j}>0\right\}$. Let the matrix of $D=\operatorname{diag}\left\{d_{i}, i=\right.$ $1,2, \ldots, n\}$, where $d_{i}=\sum_{j=1}^{n} a_{i j}$ denotes the sum of the values in $i$ th row of $A$ matrix. The matrix of $L=D-A$ presents the Laplacian matrix of graph. For the arbitrary node $i$ and node $j$, the graph $\varsigma$ is undirected graph when $a_{i j}=a_{j i}$. A directed graph is said to be strongly connected, if there is a directed path from every node to every other node. A digraph is said to have a spanning tree, if there exists a node such that there is a directed path from this node to every other node. The union of a collection of directed graphs $\varsigma_{i 1}, \varsigma_{i 2}, \ldots, \varsigma_{i j}$ with the same node set $V$ is a directed graph with node set $V$ and the edge set equal to the union of the edge sets of all of the graphs in the collection.

Given $C=\left[c_{i j}\right] \in R^{n \times r}$, it is said that $C \geq 0$ (C is nonnegative) if all its elements are nonnegative. If a nonnegative matrix $C \in R^{n \times r}$ satisfies $C \mathbf{1}_{r}=\mathbf{1}_{n}$, then it is said to be (row) stochastic. In addition, a stochastic matrix $D \in R^{n \times n}$ is said to be indecomposable and aperiodic (SIA) if $\lim _{m \rightarrow+\infty} D^{m}=$ $\mathbf{1}_{n} f^{T}$, where $f \in R^{n}$.

\section{Networks of Discrete-Time Agents with Mixed Dynamics}

In this section, we analyse networks of discrete-time multiagent with mixed dynamics, and networks consist of the first-order agents and the second-order agents. Let the total number of agents be $m+n$. Each agent is regarded as a node in the communication directed graph $\varsigma$. Each edge corresponds to an available information channel from agent $V_{i}$ to $V_{j}$ at time $k T$, where $k$ is nonnegative integer and $T>0$ is the sample time. Suppose the number of second-order agents is $m$ and the number of first-order agents is $n$. To simplify the notation, we replace all " $(k T)$ " by " $(k)$ ". Then, the dynamics of the $i$ th second-order agent are given as follows:

$$
\begin{aligned}
& x_{i}(k+1)=x_{i}(k)+v_{i}(k) T, \\
& v_{i}(k+1)=v_{i}(k)+u_{i}(k) T, \quad i=1,2, \ldots, m,
\end{aligned}
$$

where $x_{i} \in R$ is the position, $v_{i} \in R$ is the velocity, and $u_{i} \in R$ is control input. For the second-order multiagent network, it is assumed that $x_{i}(k)=x_{i}(0)$ and $v_{i}(k)=v_{i}(0)=0$ for $k<0$. Then, the dynamics of the $l$ th first-order agent are given as follows:

$$
\begin{aligned}
x_{l}(k+1)=x_{l}(k)+u_{l}(k) T, & \\
& \quad=m+1, m+2, \ldots, m+n,
\end{aligned}
$$

where $x_{l} \in R$ is the position and $u_{l} \in R$ is the control input. For the first-order multiagent network, we assume that $x_{l}(k)=x_{l}(0)$ for $k<0$.

The discrete-time multiagent networks with mixed dynamics (1) and (2) are said to reach agreement, if and only if any initial condition satisfies

$$
\begin{aligned}
& \lim _{k \rightarrow+\infty}\left(x_{i}(k)-x_{j}(k)\right)=0, \quad i, j=1,2, \ldots, m+n, \\
& \lim _{k \rightarrow+\infty}\left(v_{i}(k)-v_{j}(k)\right)=0, \quad i, j=1,2, \ldots, m .
\end{aligned}
$$

To solve the agreement problems, the protocol with time delays is proposed for the second-order agents as follows:

$$
\begin{aligned}
u_{i}(k)= & -p_{i} v_{i}(k) \\
& +\sum_{j \in N_{i}(k)} a_{i j}(k)\left(x_{j}\left(k-\tau_{i j}(k)\right)-x_{i}(k)\right) ;
\end{aligned}
$$

for the second-order multiagent network, $p_{i}>0$ is control parameter, $\tau_{i j}(k) \leq \tau_{\max }$ is the communication time delay from $v_{j}$ to $v_{i}$, and $a_{i j}(k)>0$ is the coupling weight chosen from any finite set. Define $y_{i}(k)=x_{i}(k)+v_{i}(k) T$, 
$i=1,2, \ldots, m$, and we can rewrite the dynamics of $i$ th second-order agent with algorithm (4) as follows:

$$
\begin{aligned}
& x_{i}(k+1)=y_{i}(k), \\
& \begin{aligned}
& y_{i}(k+1)=2 y_{i}(k)-x_{i}(k)+T^{2} u_{i}(k) \\
&=\left(2-p_{i} T\right) y_{i}(k)-\left(1-p_{i} T\right) x_{i}(k) \\
&+T^{2}\left(\sum_{j \in N_{i}(k)} a_{i j}(k)\left(x_{j}\left(k-\tau_{i j}(k)\right)-x_{i}(k)\right)\right), \\
& i=1,2, \ldots, m .
\end{aligned}
\end{aligned}
$$

Then, we give the agreement algorithm of the first-order agents:

$$
u_{l}(k)=\sum_{j \in N_{l}(k)} a_{l j}(k)\left(x_{j}\left(k-\tau_{l j}(k)\right)-x_{l}(k)\right) ;
$$

for the first-order multiagent network, $\tau_{l j}(k) \leq \tau_{\max }$ is the communication time delay from $v_{j}$ to $v_{l}$ and $a_{l j}(k)>0$ is the edge weight chosen from any finite set. With (6), we can rewrite the dynamics of first-order agents:

$$
\begin{aligned}
& x_{l}(k+1) \\
& =x_{l}(k) \\
& \quad+T\left(\sum_{j \in N_{l}(k)} a_{l j}(k)\left(x_{j}\left(k-\tau_{l j}(k)\right)-x_{l}(k)\right)\right), \\
& l=m+1, m+2, \ldots, m+n .
\end{aligned}
$$

In the multiagent network composed of first-order agents and second-order agents, the neighbors of each second-order agent $i$ include first-order and second-order agents, denoted by $N_{i}(k)=N_{i}^{s}(k) \cup N_{i}^{s f}(k)$, where $N_{i}^{s}(k)$ and $N_{i}^{s f}(k)$ are agent $i$ s second-order and fist-order neighboring agents, respectively. Similarly, the neighbors of each first-order agent are denoted by $N_{l}(k)=N_{l}^{f}(k) \cup N_{l}^{f s}(k)$. And we can denote the Laplacian matrix $L(k)$ as follows:

$$
L(k)=\left[\begin{array}{cc}
\bar{L}_{s}(k) & -A_{s f}(k) \\
-A_{f_{s}}(k) & \bar{L}_{f}(k)
\end{array}\right],
$$

where $\bar{L}_{s}(k)=L_{s}(k)+D_{s f}(k), L_{s}(k)$ is the Laplacian matrix of second-order agents, $D_{s f}(k)=\operatorname{diag}\left\{\sum_{j \in N_{i}^{f}} a_{i j}(k), i=\right.$ $1,2, \ldots, m\}$, and $A_{s f}(k)$ denotes the adjacency relations of second-order agents to first-order agents. Meanwhile, $\bar{L}_{f}(k)=L_{f}(k)+D_{f s}(k)$ is the Laplacian matrix of first-order agents, $D_{f_{s}}(k)=\operatorname{diag}\left\{\sum_{j \in N_{l}^{s}} a_{l j}(k), l=m+1, m+2, \ldots, m+\right.$ $n$, and $A_{f s}(k)$ denotes the adjacency relations of first-order agents to second-order agents.

\section{Main Results}

In this section, we will solve the agreement problems of networks of discrete-time agents with mixed dynamics and arbitrary bounded time delays. To analyse the stability of such multiagent networks, there are mainly three approaches: the Lyapunov-based approach, the frequency domain approach, and the approach based on the properties of nonnegative matrices. However, the frequency domain approach is limited to the fixed topology case and invalid when the topologies dynamically change, whereas the Lyapunov-based approach is hard to apply to the case of general directed graphs with time delay and switching topologies, especially when the communication graphs have no spanning trees. In this section, to use the approach based on the properties of nonnegative matrices, we performed a model transformation already (see (5)). Then, we can transform the network into an equivalent undelayed one whose network matrix is stochastic. Based on this obtained equivalent network, we present sufficient conditions under which all agents reach agreement with arbitrary bounded time delays under fixed topology and dynamically changing topologies.

Denote $\psi(k)=\left[x_{s}^{T}(k), y_{s}^{T}(k), x_{f}^{T}(k)\right]^{T}$, where $x_{s}=$ $\left[x_{1}, x_{2}, \ldots, x_{m}\right]^{T}, y_{s}=\left[y_{1}, y_{2}, \ldots, y_{m}\right]^{T}$, and $x_{f}=$ $\left[x_{m+1}, x_{m+2}, \ldots, x_{m+n}\right]^{T}$; we can rewrite the networks with mixed dynamics (5) and (7):

$$
\psi(k+1)=\Xi_{0}(k) \psi(k)+\sum_{r=1}^{\tau_{\max }} \Xi_{r} \psi(k-r),
$$

where $\Xi_{0}(k)=\operatorname{diag}(\Xi(k))$ and $\Xi_{r}(k)\left(r=0,1, \ldots, \tau_{\max }\right)$ is either zero or equal to the weight of the edge $e_{i j}$ if $\tau_{i j}=r$. Obviously, $\Xi_{r}(k)\left(r=0,1, \ldots, \tau_{\max }\right)$ are $(n+2 m) \times(n+2 m)$ matrices satisfying $\sum_{r=0}^{\tau_{\max }} \Xi_{r}(k)=\Xi(k)$, and $\Xi(k)$ is given by

$$
\begin{aligned}
& \Xi(k) \\
& =\left[\begin{array}{ccc}
0 & I_{m} & 0 \\
-I_{m}+T A-T^{2} \bar{L}_{s}(k) & 2 I_{m}-T A & T^{2} A_{s f}(k) \\
T A_{f s}(k) & 0 & I_{n}-T \bar{L}_{f}(k)
\end{array}\right],
\end{aligned}
$$

where $A=\operatorname{diag}\left\{p_{i}, i=1,2, \ldots, m\right\}$ is a diagonal matrix.

Because of the existence of time delays, it is still hard to perform analysis on network (9). We need to introduce an equivalent augmented network (9). Define a new state variable $z(k)=\left[\psi(k)^{T}, \psi(k-1)^{T}, \ldots, \psi\left(k-\tau_{\max }\right)^{T}\right]^{T}$; then network (9) can be rewritten as an undelayed network given by

$$
z(k+1)=\Phi(k) z(k)
$$

where $\Phi(k)$ is defined as

$$
\Phi(k)=\left[\begin{array}{ccccc}
\Xi_{0}(k) & \Xi_{1}(k) & \cdots & \Xi_{\tau_{\max }-1}(k) & \Xi_{\tau_{\max }}(k) \\
I & 0 & \cdots & 0 & 0 \\
0 & I & \cdots & 0 & 0 \\
\vdots & \vdots & \ddots & \vdots & \vdots \\
0 & 0 & \cdots & I & 0
\end{array}\right] .
$$


We know that $L(k) \mathbf{1}_{m+n}=0$; then it is easy to see $\Xi(k) \mathbf{1}_{2 m+n}=\mathbf{1}_{2 m+n}$. This property is important and will be used to study the stability of the networks. Before presenting the main theorem, we first introduce some necessary lemmas.

Lemma 1 (Wolfowitz, 1963 [23]). Let $p_{1}, p_{2}, \ldots, p_{n} \in R^{q \times q}$ be a finite set of SIA matrices with the property that, for each sequence $p_{i 1}, p_{i 2}, \ldots, p_{i j}$ with positive length, the matrix product $p_{i j} p_{i j-1} \cdots p_{i 1}$ is SIA. Then, for each infinite sequence $p_{i 1}, p_{i 2}, \ldots$, there exists a vector $f \in R^{q}$ such that

$$
\prod_{j=1}^{\infty} p_{i j}=\mathbf{1}_{n} f^{T}
$$

Lemma 2 (Ren and Beard, 2005 [11]). Given a matrix $A=$ $\left[a_{i j}\right] \in M_{n}(R)$, where $a_{i i} \leq 0, a_{i j} \geq 0$, for all $i \neq j$, and $\sum_{j=1}^{n} a_{i j}=0$ for each $j$, then $A$ has at least one zero eigenvalue and all of the nonzero eigenvalues are in the open left halfplane. Furthermore, A has exactly one zero eigenvalue if and only if the directed graph associated with $A$ has a spanning tree.

Lemma 3 (Ren and Beard, 2005 [11]). Let $A=\left[a_{i j}\right] \in M_{n}(R)$ be a stochastic matrix. If $A$ has an eigenvalue $\lambda=1$ with algebraic multiplicity equal to one, and all the other eigenvalues satisfy $|\lambda|<1$, then $A$ is SIA; that is, $\lim _{m \rightarrow \infty} A^{m} \rightarrow \mathbf{1}_{n} v^{T}$, where $v$ satisfies $A^{T} v=v$ and $\mathbf{1}_{n}^{T} v=\mathbf{1}_{n}$. Furthermore, each element of $v$ is nonnegative.

To lead to the following lemmas and theorems and to be satisfied with some properties of matrix, we give an assumption that

$$
\begin{gathered}
1+T^{2} \sum_{j \in N_{i}(k)} a_{i j}(k)<T p_{i}<2, \\
T \sum_{j \in N_{l}(k)} a_{l j}(k)<1,
\end{gathered}
$$

for $i=1, \ldots, m$ and $l=m+1, \ldots, m+n$.

Lemma 4. Under assumption (14), we can know that $\Xi(k)$ is a stochastic matrix and $\Phi(k)$ is also a stochastic matrix.

Proof. It is clear that all elements of $\Xi(k)$ are nonnegative under assumption (14), and $\Xi(k) \mathbf{1}_{2 m+n}=\mathbf{1}_{2 m+n}$. Thus, $\Xi(k)$ is a stochastic matrix. Similarly, we can know that $\Phi(k)$ defined in (12) is also a stochastic matrix.

Lemma 5. With assumption (14), if the interconnection topology $\varsigma$ of $m+n$ agents (5) and (7) has a spanning tree, the matrix $\Phi(k)$ defined in (12) is SIA.
Proof. Taking the elementary column transform of $\widehat{\Phi}(k)=$ $\Phi(k)-I_{\left(\tau_{\max }+1\right)(n+2 m)}$ by adding all the other columns to the last column,

$$
\begin{aligned}
& \widehat{\Phi}(k) \\
& =\left[\begin{array}{ccccc}
\Xi_{0}(k)-I & \Xi_{1}(k) & \cdots & \Xi_{\tau_{\max }-1}(k) & \Xi(k)-I \\
I & -I & \cdots & 0 & 0 \\
0 & I & \cdots & 0 & 0 \\
\vdots & \vdots & \ddots & \vdots & \vdots \\
0 & 0 & \cdots & I & 0
\end{array}\right],
\end{aligned}
$$

we can know that $\operatorname{rank}(\widehat{\Phi}(k))=\tau_{\max }(n+2 m)+\operatorname{rank}(\Xi(k)-$ $\left.I_{n+2 m}\right)$, where $\Xi(k)$ is defined in (10). Similarly, take the elementary column and row transforms of $\widehat{\Xi}(k)=\Xi(k)-$ $I_{n+2 m}$. First, exchange the first and the second column. Then, add the first column to the second column. Finally, the second row divides $T^{2}$ and the third row divides $T$. We can obtain $\widehat{\Xi}(k)=\Xi(k)-I_{n+2 m}$ as follows:

$$
\widehat{\Xi}(k) \longrightarrow\left[\begin{array}{ccc}
I_{m} & 0 & 0 \\
\frac{I_{m}}{T^{2}}-\frac{A}{T} & -\bar{L}_{s}(k) & A_{s f}(k) \\
0 & A_{f s}(k) & -\bar{L}_{f}(k)
\end{array}\right],
$$

so $\operatorname{rank}(\widehat{\Xi}(k))=\operatorname{rank}(L(k))+m$. Thus, if and only if $\operatorname{rank}(L(k))=m+n-1, \Phi(k)$ has an eigenvalue $\lambda=1$ with the algebraic multiplicity equal to one; that is, the topology of agents (5) and (7) has a spanning tree from Lemma 2. Similarly, by taking the elementary column transform, we can prove that $\operatorname{rank}\left(\Phi+I_{\left(\tau_{\max }+1\right)(n+2 m)}\right)=\left(\tau_{\max }+1\right)(n+2 m)$ is full rank, which holds under arbitrary topology with assumption (14); that is, it has $\lambda \neq-1$. Based on the properties of stochastic matrix and Gershgorin disk theorem, except for one eigenvalue $\lambda=1$, all the other eigenvalues satisfy $|\lambda|<1$. Thus, $\Phi(k)$ is SIA based on Lemma 3.

Theorem 6. Consider the discrete-time multiagent networks with mixed dynamics (5) and (7) and bounded time delays under fixed topology. In addition, $a_{i j}(k)$ and $a_{l j}(k)$ do not change with time. Under assumption (14), if the digraph $\varsigma$ of $m+$ $n$ agents has a spanning tree, the agents in (5) and (7) can reach stationary agreement with protocols (4) and (6).

Proof. We can rewrite network (11) as $z(k+1)=\Phi(k)^{k+1} z(0)$. Because fixed topology has a spanning tree, we can obtain that $\Phi(k)$ is SIA from Lemma 5. Then, $\lim _{k \rightarrow \infty} z(k)=$ $\mathbf{1}_{(n+2 m)\left(\tau_{\max }+1\right)} f^{T} z(0)$ from Lemma 1, where some vector $f \in$ $R^{(n+2 m)\left(\tau_{\max }+1\right)}$. Thus, $\lim _{k \rightarrow \infty} x_{i}(k)=f^{T} z(0), i=1,2, \ldots, m+$ $n$; that is, the agents in (5) and (7) reach stationary agreement.

Lemma 7. Under assumption (14), if the union of the digraphs $\varsigma\left(\gamma_{1}\right), \varsigma\left(\gamma_{1}+1\right), \ldots, \varsigma\left(\gamma_{2}\right)$ of agents (5) and (7) has spanning trees for the positive integers $\gamma_{1}$ and $\gamma_{2}$ with $\gamma_{1}<\gamma_{2}$, then $\prod_{\gamma_{1}}^{\gamma_{2}} \Phi(k)$ is SIA. 
Proof. We define the union of the digraphs associated with $I_{n+2 m}-\Xi\left(\gamma_{1}\right), I_{n+2 m}-\Xi\left(\gamma_{1}+1\right), \ldots, I_{n+2 m}-\Xi\left(\gamma_{2}\right)$ as $\widetilde{\varsigma}\left(\gamma_{1}\right), \widetilde{\varsigma}\left(\gamma_{1}+\right.$ $1), \ldots, \widetilde{\varsigma}\left(\gamma_{2}\right)$. Under assumption (14), if the union of the digraphs $\varsigma\left(\gamma_{1}\right), \varsigma\left(\gamma_{1}+1\right), \ldots, \varsigma\left(\gamma_{2}\right)$ of agents (5) and (7) has a spanning tree, then the matrix $\left(1 /\left(\gamma_{2}-\gamma_{1}\right)\right)\left(\sum_{\gamma_{1}}^{\gamma_{2}} \Xi(k)\right)$ has an eigenvalue $\lambda=1$ with the algebraic multiplicity equal to one from the proof of Lemma 5; that is, the rank of matrix $\left(I_{n+2 m}-\left(1 /\left(\gamma_{2}-\gamma_{1}\right)\right)\left(\sum_{\gamma_{1}}^{\gamma_{2}} \Xi(k)\right)\right)$ is $n+2 m-1$. Then, we can obtain that the digraph of the Laplacian matrix $I_{n+2 m}-\left(1 /\left(\gamma_{2}-\right.\right.$ $\left.\left.\gamma_{1}\right)\right)\left(\sum_{\gamma_{1}}^{\gamma_{2}} \Xi(k)\right)$ has a spanning tree from Lemma 2 ; that is, the union of the digraphs $\widetilde{\varsigma}\left(\gamma_{1}\right), \widetilde{\varsigma}\left(\gamma_{1}+1\right), \ldots, \widetilde{\varsigma}\left(\gamma_{2}\right)$ has a spanning tree. Then, by using a similar proof for Lemma (7) in Xiao and Wang (2006) [21], Lemma 7 can be proved.

Theorem 8. Under assumption (14), if there exists an infinite sequence of uniformly bounded, nonoverlapping time intervals $\left[\gamma_{l}, \gamma_{l+1}\right), 0<\gamma_{l+1}-\gamma_{l}<\alpha, \alpha, l \in Z_{+}$, such that the union of the digraphs of $n$ agents across each interval $\left[\gamma_{l}, \gamma_{l+1}\right)$ has a spanning tree, then multiagent networks (5) and (7) reach agreement with protocols (4) and (6).

Proof. For each $k \geq 0$, let $l_{k}$ be the largest integer such that $k_{l_{k}} \leq k$. Then, network (11) is rewritten as

$$
z(k+1)=\Phi(k) \cdots \Phi\left(k_{l_{k}}\right) \prod_{m=0}^{l_{k}-1} \Omega(m) z(0),
$$

where $\Omega(l)=\Phi\left(k_{l+1}-1\right) \Phi\left(k_{l+1}-2\right) \cdots \Phi\left(k_{l}\right)$. Since the union of $n$ agents interconnection topologies across $\left[k_{l}, k_{l+1}\right)$ has a spanning tree, $\Omega(l)$ is SIA from Lemma 7. Since $0<$ $\gamma_{l+1}-\gamma_{l}<\alpha$ and the edge weights $a_{i j}(k)$ are chosen from any finite set, then it is easy to see that the set of all possible $\Omega(l)$ is finite. Hence, by Lemma 7 again, we know that $\prod_{m=1}^{\infty} \Omega(m)=$ $\mathbf{1}_{(n+2 m)\left(\tau_{\max }+1\right)} f^{T}$ for some vector $f \in R^{(n+2 m)\left(\tau_{\max }+1\right)}$. We know that $\Phi(k)$ is a stochastic matrix from Lemma 4 , so we can obtain

$$
\lim _{k \rightarrow \infty} z(k+1)=\mathbf{1}_{(n+2 m)\left(\tau_{\max }+1\right)} f^{T} z(0) .
$$

Thus, $\lim _{k \rightarrow \infty} x_{i}(k)=f^{T} z(0), i=1,2, \ldots, m+n$. That is, the stationary agreement of agents (5) and (7) is reached.

Remark 9. In this section, with assumption (14), we just give the sufficient agreement criteria in Theorems 6 and 8. Similar to the first-order and the second-order multiagent networks, the multiagent networks with mixed dynamics and bounded time delays can also achieve an asymptotic agreement with fixed topologies or dynamically changing topologies.

Remark 10. Under assumption (14), we obtain two conclusions about fixed topology and dynamically changing topology with bounded time delays. First, the multiagent networks with mixed dynamics (1) and (2) and fixed topology achieve stationary agreement using protocol (4) and (6) if and only if fixed topology has a spanning tree. Second, the multiagent networks with mixed dynamics (1) and (2) and dynamically changing topologies achieve stationary agreement using protocols (4) and (6) that any of the changing topologies may not

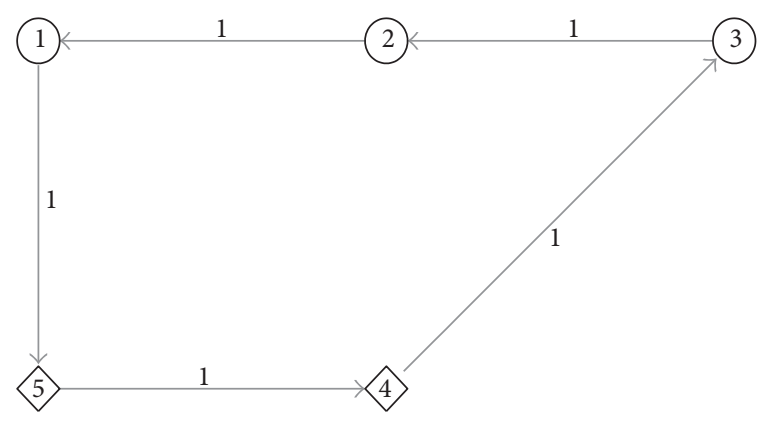

Figure 1: Topology.

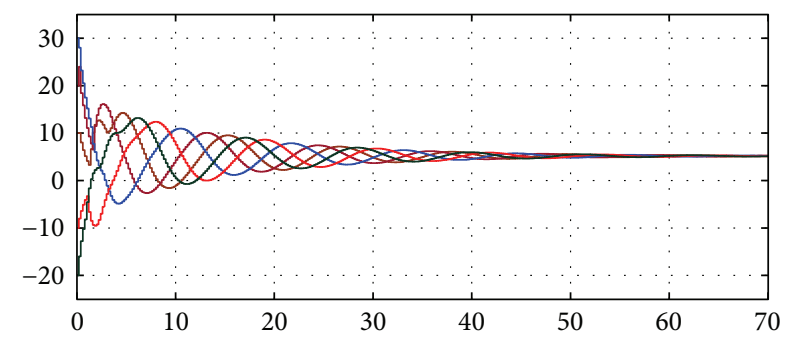

FIgURe 2: Position of the dynamic agents.

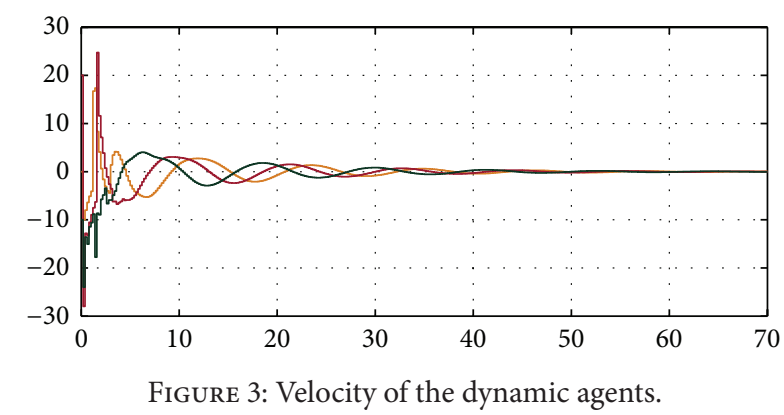

have spanning trees, but the union of the changing topologies must have a spanning tree.

\section{Simulations}

We suppose a multiagent network composed of three secondorder agents (5) and two first-order agents (7). The interconnection topology of the network has a spanning tree in Figure 1. First, we simulate the network with fixed topology. In fixed topology, the diamonds denote the first-order agents and the circles denote the second-order agents. The sampling time of networks is $T=0.2 \mathrm{~s}$. The weight of each edge in topology is 1 and the control parameters $p_{1}=6, p_{2}=7$, and $p_{3}=8$ for the second-order dynamic agents. Besides, the communication time delays associated with topology are $\tau_{12}=3, \tau_{51}=4, \tau_{45}=5, \tau_{34}=6$, and $\tau_{23}=7$. Then, assumption (14) is satisfied. The initial assumptions are $x(0)=$ $[10,20,30,-10,-20]^{T}, v(0)=[0,20,-10]^{T}$. Thus, stationary agreement of the agents in (5) and (7) can be achieved (see Figures 2 and 3). Then, we simulate the network with the 


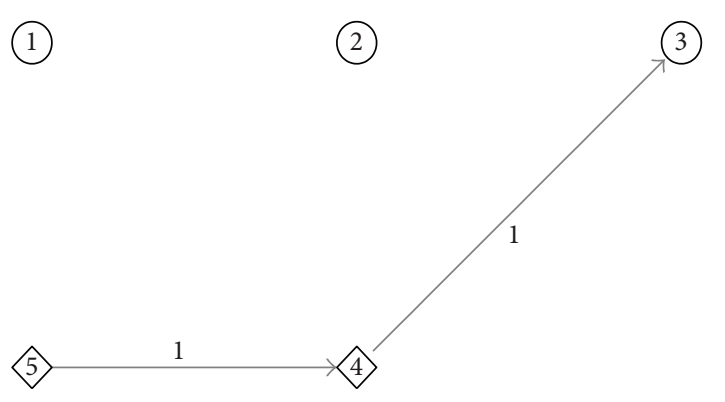

(a) $\mathrm{Ga}$

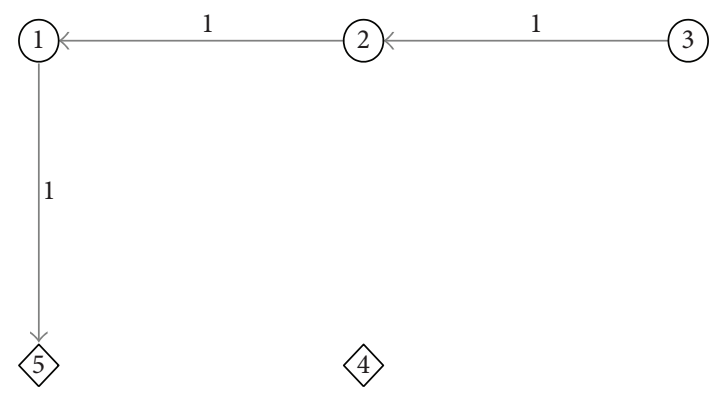

(b) $\mathrm{Gb}$

FIGURE 4: Two directed graphs.
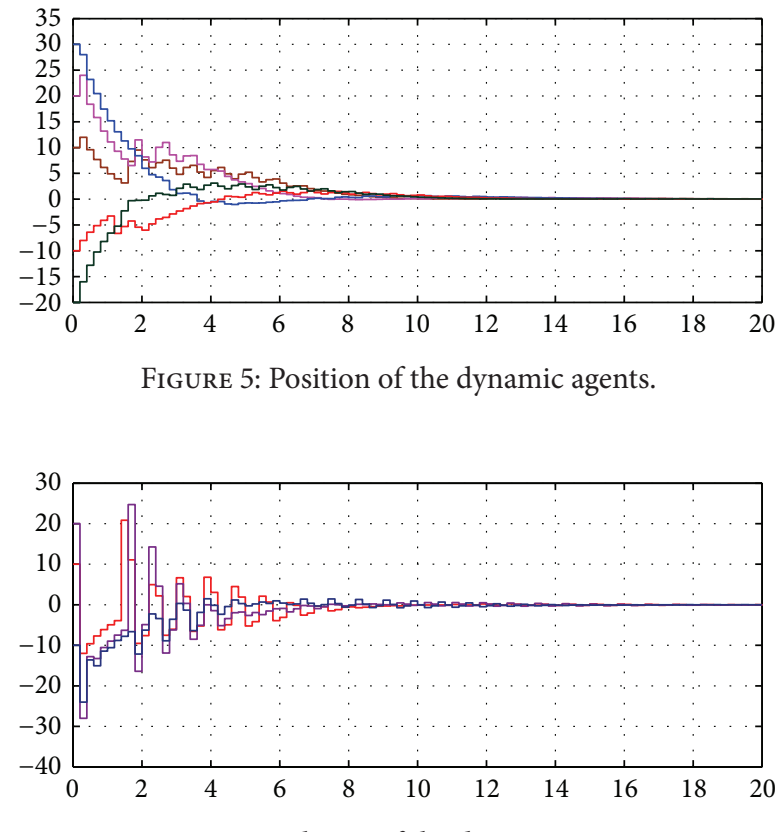

FIGURE 6: Velocity of the dynamic agents.

dynamically changing topologies. In Figure 4, a finite state machine is shown with two states, $\mathrm{Ga}$ and $\mathrm{Gb}$, which denote the states of a network with dynamically changing topology and time delay; it starts at Ga and switches every $2 T$ s to the next state. Note that in each time interval of $4 T \mathrm{~s}$ the union of the communication graphs $\mathrm{Ga} \cup \mathrm{Gb}$ has a spanning tree. Let the other parameters be similar to fixed topology; then assumption (14) is satisfied, and agreement is achieved as shown in Figures 5 and 6, which is consistent with Theorem 8.

According to Figure 1, we can obtain the Laplacian matrix

$$
L=\left[\begin{array}{ccccc}
1 & 0 & 0 & 0 & -1 \\
-1 & 1 & 0 & 0 & 0 \\
0 & -1 & 1 & 0 & 0 \\
0 & 0 & -1 & 1 & 0 \\
0 & 0 & 0 & -1 & 1
\end{array}\right]
$$

\section{Conclusion}

This paper studies the agreement problems of networks of discrete-time agents with mixed dynamics and arbitrary bounded time delays under fixed topology and dynamically changing communication topologies. For discrete-time multiagent networks with mixed dynamics, by using model transformations and the properties of nonnegative matrix, if and only if fixed topology has a spanning tree, the agents with some restriction on the coupling weights and the sampling interval can tolerate arbitrary bounded time delays to reach a stationary agreement. However, the multiagent networks with mixed dynamics and arbitrary bounded time delays under dynamically changing topologies achieve stationary agreement that any of the changing topologies may not have spanning trees, but the union of the changing topologies must have a spanning tree.

\section{Conflict of Interests}

The authors declare that there is no conflict of interests regarding the publication of this paper.

\section{Acknowledgments}

This work was supported by the National Natural Science Foundation of China (61203080, 61573082), the Foundation of State Key Laboratory of Networking and Switching Technology Foundation (SKLNST2011105, SKLNST2013109), National Program 863 of China (2014AA4032), National Program 973 of China (613237201506), and State Key Laboratory of Intelligent Control and Decision of Complex Systems.

\section{References}

[1] Y. Zheng, Y. Zhu, and L. Wang, "Consensus of heterogeneous multi-agent systems," IET Control Theory \& Applications, vol. 5, no. 16, pp. 1881-1888, 2011.

[2] L. Moreau, "Stability of continuous-time distributed consensus algorithms," in Proceedings of the 43rd IEEE Conference on Decision and Control (CDC '04), pp. 3998-4003, IEEE, December 2004 . 
[3] Y. Hong, L. Gao, D. Cheng, and J. Hu, "Lyapunov-based approach to multiagent systems with switching jointly connected interconnection," IEEE Transactions on Automatic Control, vol. 52, no. 5, pp. 943-948, 2007.

[4] P. Lin, Y. Jia, and L. Li, "Distributed robust $H_{\infty}$ consensus control in directed networks of agents with time-delay," Systems \& Control Letters, vol. 57, no. 8, pp. 643-653, 2008.

[5] H. Sayyaadi and M. R. Doostmohammadian, "Finite-time consensus in directed switching network topologies and timedelayed communications," Scientia Iranica, vol. 18, no. 1, pp. 7585, 2011.

[6] P. Lin and Y. Jia, "Consensus of a class of second-order multiagent systems with time-delay and jointly-connected topologies," IEEE Transactions on Automatic Control, vol. 55, no. 3, pp. 778-784, 2010.

[7] P. Lin and Y. Jia, "Multi-agent consensus with diverse timedelays and jointly-connected topologies," Automatica, vol. 47, no. 4, pp. 848-856, 2011.

[8] W. Zhang, J. Liu, D. Zeng, and T. Yang, "Consensus analysis of continuous-time second-order multi-agent systems with nonuniform time-delays and switching topologies," Asian Journal of Control, vol. 15, no. 5, pp. 1516-1523, 2013.

[9] P. Lin and Y. Jia, "Consensus of second-order discrete-time multi-agent systems with nonuniform time-delays and dynamically changing topologies," Automatica, vol. 45, no. 9, pp. 21542158, 2009.

[10] P. Lin and W. Ren, "Constrained consensus in unbalanced networks with communication delays," IEEE Transactions on Automatic Control, vol. 59, no. 3, pp. 775-781, 2014.

[11] W. Ren and R. W. Beard, "Consensus seeking in multiagent systems under dynamically changing interaction topologies," IEEE Transactions on Automatic Control, vol. 50, no. 5, pp. 655661, 2005.

[12] B. Liu, H. Su, R. Li, D. Sun, and W. Hu, "Switching controllability of discrete-time multi-agent systems with multiple leaders and time-delays," Applied Mathematics and Computation, vol. 228, pp. 571-588, 2014.

[13] P. Lin and Y. Jia, "Distributed rotating formation control of multi-agent systems," Systems \& Control Letters, vol. 59, no. 10, pp. 587-595, 2010.

[14] M. Lipo, C. Xianbing, and Z. Hongbo, "Robust optimal coordination for multiagent systems with uncertainty and time-delay," in Proceedings of the 3th International Conference on Computer and Net-Work Technology, pp. 403-409, 2011.

[15] M. Lipo and J. Yingmin, " $\mathrm{H}_{\infty}$ consensus of a class of high-order multi-agent systems with time-delay," IET Control Theory and Application, vol. 5, no. 1, pp. 247-253, 2011.

[16] W. Zhang, D. Zeng, Y. Niu, and T. Chang, "Robust consensus of second-order multi-agent systems with nonuniform timedelays and switching topologies," in Proceedings of the 19th International Conference on Methods and Models in Automation and Robotics (MMAR '14), pp. 758-763, IEEE, Miedzyzdroje, Poland, September 2014.

[17] P. Lin, W. Ren, and Y. Song, "Distributed multi-agent optimization subject to nonidentical constraints and communication delays," Automatica, vol. 65, pp. 120-131, 2016.

[18] R. Olfati-Saber and R. M. Murray, "Consensus problems in networks of agents with switching topology and time-delays," IEEE Transactions on Automatic Control, vol. 49, no. 9, pp. 15201533, 2004.
[19] C.-L. Liu and F. Liu, "Stationary consensus of heterogeneous multi-agent systems with bounded communication delays," Automatica, vol. 47, no. 9, pp. 2130-2133, 2011.

[20] S. Yi-Jie, Z. Guo-liang, and Z. Jing, "Consensus analysis for a class of heterogeneous multiagent systems with time delay based on frequency domain method," Mathematical Problems in Engineering, vol. 2014, Article ID 248684, 7 pages, 2014.

[21] F. Xiao and L. Wang, "State consensus for multi-agent systems with switching topologies and time-varying delays," International Journal of Control, vol. 79, no. 10, pp. 1277-1284, 2006.

[22] W. Ren, R. W. Beard, and T. W. McLain, "Coordination variables and consensus building in multiple vehicle systems," Cooperative Control, vol. 309, pp. 171-188, 2005.

[23] J. Wolfowitz, "Products of indecomposable, aperiodic, stochastic matrices," Proceedings of the American Mathematical Society, vol. 14, no. 5, pp. 733-737, 1963. 


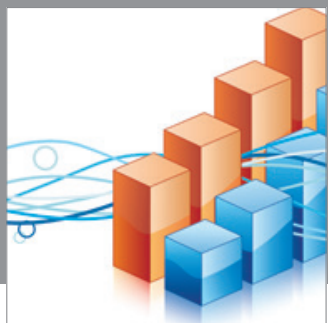

Advances in

Operations Research

mansans

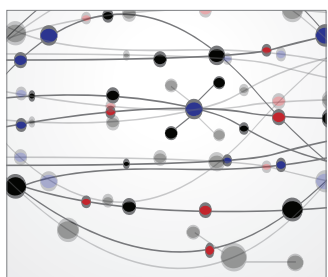

The Scientific World Journal
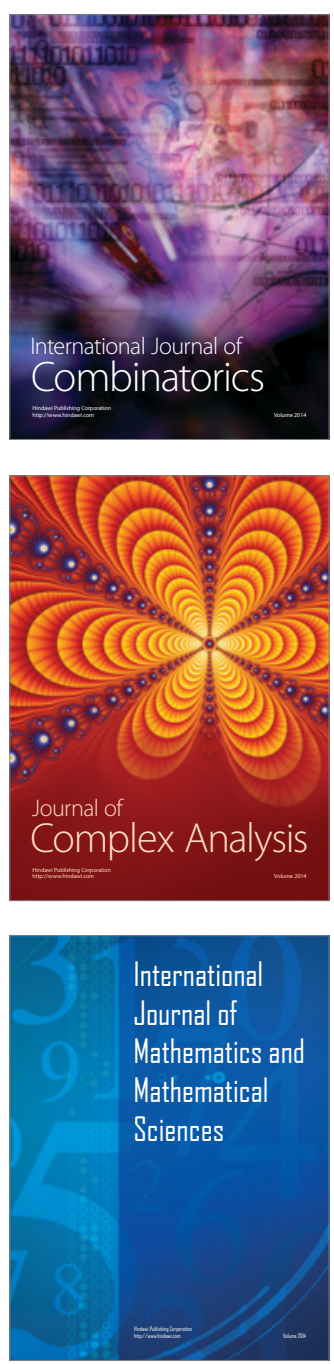
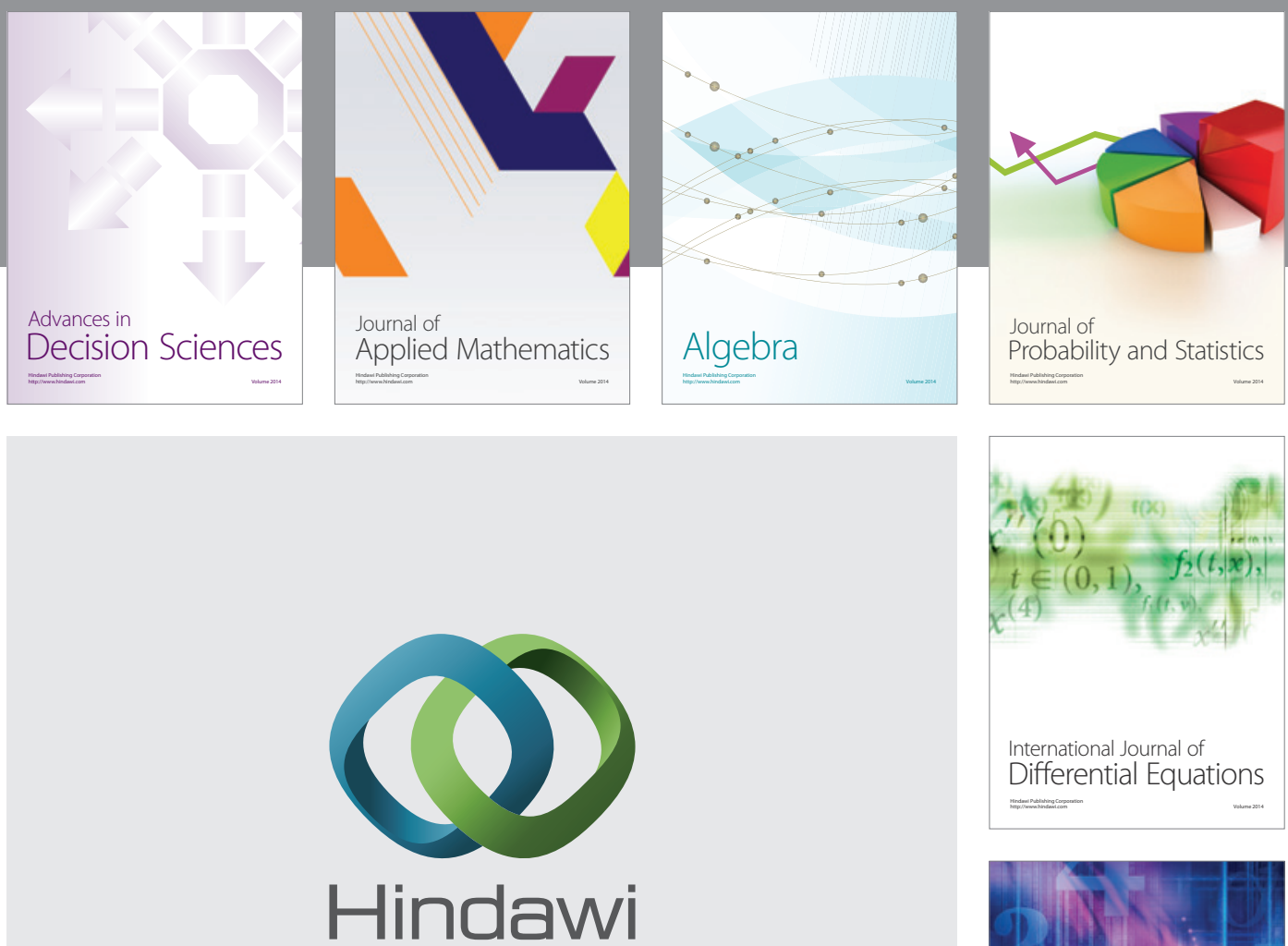

Submit your manuscripts at http://www.hindawi.com
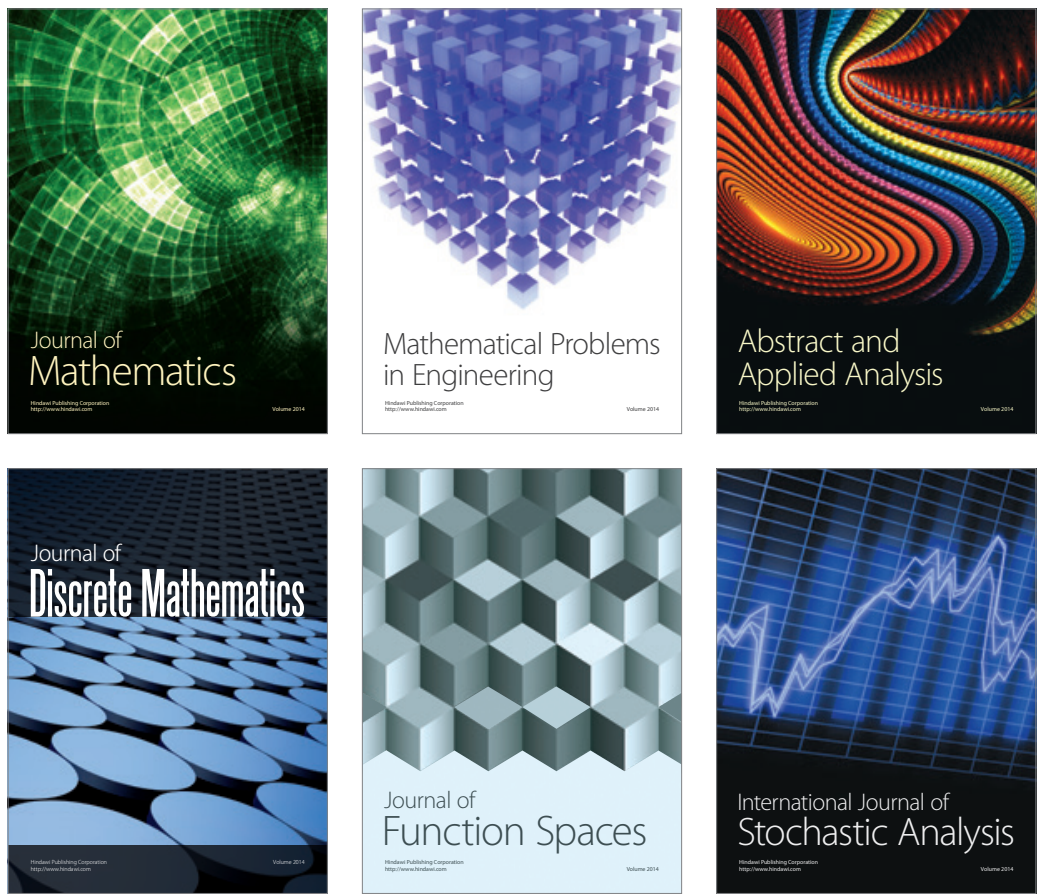

Journal of

Function Spaces

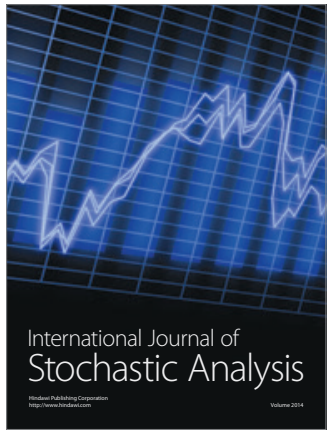

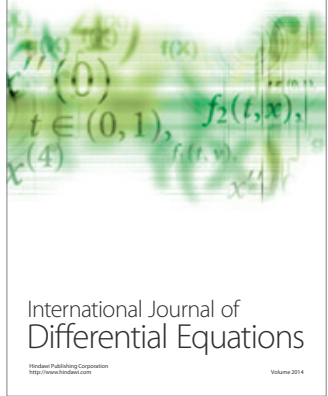
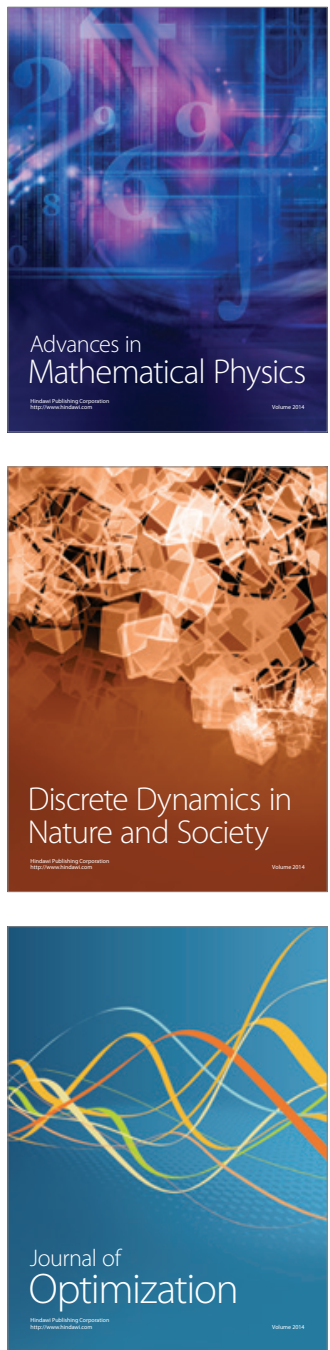